\title{
Six-month decline of serum anti-spike S1 subunit IgA in SARS-CoV-2 in seronegative healthcare workers after mRNA-based COVID-19 vaccination
}

\section{Gian Luca Salvagno}

Section of Clinical Biochemistry, University of Verona, Verona, Italy and Service of Laboratory Medicine, Pederzoli Hospital, Peschiera del Garda, Italy

\section{Brandon M. Henry}

Clinical Laboratory, Division of Nephrology and Hypertension, Cincinnati Children's Hospital Medical Center, Cincinnati, $\mathrm{OH}$, USA and Host-Pathogens Interactions and Population Health Programs, Texas Biomedical Research Institute, San Antonio, Texas, USA

\section{Laura Pighi}

Section of Clinical Biochemistry, University of Verona, Verona, Italy and Service of Laboratory Medicine, Pederzoli Hospital, Peschiera del Garda, Italy

\section{Simone De Nitto}

Section of Clinical Biochemistry, University of Verona, Verona, Italy and Service of Laboratory Medicine, Pederzoli Hospital, Peschiera del Garda, Italy

Giuseppe Lippi ( $\sim$ giuseppe.lippi@univr.it)

Section of Clinical Biochemistry, University of Verona, Verona, Italy

\section{Short Report}

Keywords: COVID-19, SARS-CoV-2, Vaccination, Antibodies, Immune response

Posted Date: October 26th, 2021

DOI: https://doi.org/10.21203/rs.3.rs-1019658/v1

License: (c) (1) This work is licensed under a Creative Commons Attribution 4.0 International License. Read Full License 


\section{Six-month decline of serum anti-spike S1 subunit IgA in SARS-CoV-2 in seronegative healthcare workers after mRNA-based COVID-19 vaccination}

\section{Gian Luca Salvagno $^{1,2}$, Brandon M. Henry ${ }^{3,4}$, Laura Pighi ${ }^{1,2}$, Simone De Nitto ${ }^{1,2}$, Giuseppe Lippi ${ }^{1}$}

1. Section of Clinical Biochemistry, University of Verona, Verona, Italy

2. Service of Laboratory Medicine, Pederzoli Hospital, Peschiera del Garda, Italy

3. Clinical Laboratory, Division of Nephrology and Hypertension, Cincinnati Children's Hospital Medical Center, Cincinnati, OH, USA

4. Host-Pathogens Interactions and Population Health Programs, Texas Biomedical Research Institute, San Antonio, Texas, USA

Short title: Anti-spike S1 subunit IgA kinetic after vaccination

Keywords: COVID-19; SARS-CoV-2; Vaccination; Antibodies; Immune response

Word count: $1205+2$ Figures

Corresponding author:

Prof. Giuseppe Lippi

Section of Clinical Biochemistry

University Hospital of Verona

Piazzale L.A. Scuro, 10

37134 Verona - Italy

Tel. 0039-045-8122970

Fax. 0039-045-8124308

Email: giuseppe.lippi@univr.it 


\section{ABSTRACT}

Background: Since serum anti-SARS-CoV-2 (severe acute respiratory syndrome coronavirus 2) IgA antibodies correlate with secretory anti-SARS-CoV-2 IgA and contribute to virus neutralization, we planned an observational study to measure serum anti-SARS-CoV-2 IgAs kinetics throughout a 6-month period in coronavirus disease 2019 (COVID-19) vaccine recipients.

Methods: The study sample consisted of 97 baseline SARS-CoV-2 seronegative healthcare workers (median age 42 years and IQR 31-52 years; 52 females), who underwent vaccination with Pfizer/BioNTech Comirnaty mRNA-based vaccine (two 30 $\mu \mathrm{g}$ doses, 21 days apart). Serum samples were collected at baseline, before the second vaccine dose (i.e., day 21), and then 51, 111 and 201 days after enrolment (i.e., 1, 3 and 6 months after the second vaccine dose). Serum anti-SARS-CoV-2 spike S1 subunit IgA were measured with Anti-SARS-CoV-2 ELISA IgA (Euroimmun, Lübeck, Germany).

Results: Anti-SARS-CoV-2 spike S1 subunit IgA displayed a peak at 1 month after the second vaccine dose, but then progressively waned afterwards. The 6-month serum antispike $\mathrm{S} 1$ subunit $\operatorname{IgA}$ concentration was $71 \%$ lower than the peak concentration. The rate of subjects with positive $\operatorname{IgA}$ values was $0 \%$ at baseline, $80.4 \%$ at day $21,97.9 \%$ at day 51 , but then declined to $73.2 \%$ and $53.6 \%$ at 3 and 6 months after the second vaccine dose. Serum anti-spike S1 subunit IgAs measured at 111 and 201 days was significantly lower than at the 51-day peak (both $\mathrm{p}<0.001$ ). Significant inverse correlation was found between anti-SARS-CoV-2 IgA antibodies decline at 6 months/ and recipients' age $(\mathrm{r}=-0.24 ; \mathrm{p}=0.019)$.

Conclusion: These findings may provide possible explanation to decreased efficacy of COVID-19 vaccines in preventing SARS-CoV-2 infection 6 months after vaccination. 


\section{Introduction}

Widespread vaccination is now considered the mainstay for limiting the dramatic medical, social and economic consequences of the ongoing coronavirus disease 2019 (COVID-19) pandemic together with the use of physical preventive measures such as face masking, social distancing, and hand hygiene [1]. Several lines of evidence now attest that the immediate efficacy of most of the currently available COVID-19 vaccines is considerably high (i.e., over 90\%) against the risk of hospitalization, severe COVID-19 illness and death, whilst effectiveness against the risk

of infection is lower, typically comprised between 70-90\% [2]. Another important aspect that has been recently highlighted, is that vaccine efficacy against the risk of developing severe COVID-19 illness seems only marginally reduced after 6 months, whilst the efficacy for preventing SARS-CoV-2 (severe acute respiratory syndrome coronavirus 2) infection displays a much faster and sharper decline. For example, two recent US-based studies estimated that vaccine efficacy against hospitalization in people who received the Moderna or Pfizer/BioNTech mRNA-based vaccines remained substantially unvaried after 6 months, while the efficacy in preventing any type of SARS-CoV-2 infection declined between 10 to over $20 \%$, depending on the population studied [3,4]. A faster decline of mucosal humoral immunity is a possible explanation for such discrepancy, since secretary immunoglobulins, especially immunoglobulin of the IgA class, represent one of the first barriers against respiratory pathogens. Since it has been demonstrated that anti-SARS-CoV-2 IgAs contribute to higher virus neutralization on mucosal surfaces compared with IgGs, and a high correlation has also been demonstrated between serum and secretory anti-SARS-CoV-2 IgA [5], we planned this observational study to measure serum IgAs kinetics throughout a 6-month period in recipients of the Pfizer/BioNTech COVID-19 vaccine. 


\section{Materials and Methods}

The study population consisted of 100 consecutive SARS-CoV-2 seronegative healthcare workers of Peschiera del Garda hospital (Italy), who underwent a full cycle of vaccination (i.e., two $30 \mu \mathrm{g}$ doses, 21 days apart) with the Pfizer/BioNTech Comirnaty mRNA-based vaccine (Pfizer Inc., New York, NY, US). Serum samples were collected at baseline (i.e., before the first vaccine dose), immediately before the second vaccine dose (i.e., day 21), and then 51, 111 and 201 days after enrolment (i.e., 1,3 and 6 months after the second vaccine dose). Baseline seronegative status was defined as a negative result of anti-spike receptor binding domain (RBD) total serum antibodies, measured with Elecsys Anti-SARS-CoV-2 S immunoassay on Roche Cobas 6000 (Roche Diagnostics, Basel, Switzerland). The serum anti-SARS-CoV-2 spike S1 subunit IgA were measured with Anti-SARS-CoV-2 ELISA IgA (Euroimmun, Lübeck, Germany), a manual ELISA whose analytical characteristics and diagnostic performance have been comprehensively reported elsewhere [6,7]. Briefly, the overall imprecision was found to be $<2 \%$ [6], the cumulative negative and positive predictive values as high as $98.7 \%$ and $66.3 \%$, with $100 \%$ sensitivity compared with a cell culturebased microneutralization test [7]. The result of this test is positive when the value (expressed as ratio to the cut-off) is $\geq 1.1$. Results of measurements were expressed as median and interquartile range (IQR), and analyzed with Mann-Whitney U and Chisquare (with Yates' correction) tests, and with Spearman's correlation. All study subjects provided written informed consent for being vaccination and for undergoing anti-SARS-CoV-2 antibodies monitoring. The study was conducted in accordance with the Helsinki Declaration and cleared by the Ethics Committee of Verona and Rovigo Provinces (3246CESC).

\section{Results}


Our final study sample consisted of 97 baseline SARS-CoV-2 seronegative healthcare workers (median age 42 years and IQR 31-52 years; 52 females), since 3 subjects were lost on follow-up. The kinetics of serum anti-spike S1 subunit IgA is shown in figure 1, demonstrating that $\operatorname{IgA}$ antibodies concentration displays a clear peak at 1 month after the second vaccine dose, but then progressively wanes afterwards. In particular, the 6-month serum anti-spike S1 subunit IgA concentration was $71 \%$ lower (95\% CI, -81 to $-61 \%$ ) than the corresponding concentration at the peak. The rate of subjects with positive serum anti-spike $\mathrm{S} 1$ subunit $\operatorname{IgA}$ values (i.e., $\geq 1.1$ ratio) was 0/97 $(0 \%)$ at baseline, $78 / 97(80.4 \%)$ at day $21,95 / 97(97.9 \%)$ at day 51 , but then gradually declined to 71/97 (73.2\%) and 52/97 (53.6\%) at days 111 and 201 (i.e., 3 and 6 months after the second vaccine dose), respectively. The serum anti-spike S1 subunit IgA concentration measured at both 111 and 201 days was significantly lower than that measured at the 51-day peak (both $\mathrm{p}<0.001$ ) (Fig. 1). The rate of subjects with negative anti-spike $\mathrm{S} 1$ subunit IgA values (i.e., <1.1 ratio) after 6 months did not differ between males and females (51.1\% vs. 55.8\%; $\mathrm{p}=0.065)$, but was slightly higher among subjects aged $\geq 60$ years $(60.0 \%$ vs. $52.9 \% ; \mathrm{p}=0.009)$. A significant inverse correlation was found between the ratio of antibodies decline measured at 6 months and at the peak (i.e., [201day/51-day concentration]) and the age of vaccine recipients ( $r=-0.24 ; 95 \% \mathrm{CI},-0.42$ to -0.04; $\mathrm{p}=0.019$ ) (Fig. 2). The median decline was found to be virtually identical between men and women $(-72$ vs. $-71 \% ; \mathrm{p}=0.161)$.

\section{Discussion}

The efficacy of COVID-19 vaccination can be evaluated by a number of different endpoints, such as prevention of any type of SARS-CoV-2 infection, lower viral load, prevention of symptomatic disease, as well as prevention of hospitalization, mechanical ventilation, intensive care and death [8]. Recent evidence suggests that the 
efficacy of the currently available COVID-19 vaccines remains relatively high against the most severe endpoints, though they seem to display a much faster decline of effectiveness against asymptomatic or mildly symptomatic SARS-CoV-2 infection. Although we would all agree that preventing severe illness and therefore averting pressure on healthcare systems is the primary goal of vaccination, preventing infections and hence limiting viral spread is not a negligible goal, as continuous circulation of the virus may foster the emergence of new variants of concern, which may decrease further the efficacy of adaptive immunity acquired by either infection or vaccination [9]. Accordingly, the identification of potential surrogates of waned immunity and protection is especially important for purposes of targeted booster vaccination.

Although IgA assessment in serum cannot be straightforwardly associated with mucosal immunity, a high correlation has been demonstrated between serum and secretory anti-SARS-CoV-2 IgA by Sterlin et al. (i.e., $r=0.796$ ) [5], as well as between anti-spike S1 subunit IgA and neutralizing antibodies titer by Varnaitè and colleagues [10]. A high correlation (i.e., $r=0.606$ ) has also been observed by Lapić et al. between anti-SARS-CoV-2 RBD (receptor binding domain) antibody levels in paired samples of saliva and serum of Pfizer/BioNTech mRNA vaccine recipients [11]. Therefore, serum anti-SARS-CoV-2 IgA concentration could be considered an acceptable surrogate of mucosal humoral immunity [12]. The results of our study demonstrate that the serum levels of anti-spike S1 subunit IgA consistently declined 6 months after Pfizer/BioNTech mRNA-based vaccine administration in healthcare workers, with nearly $50 \%$ of them undergoing $\operatorname{Ig} \mathrm{A}$ seronegativization. Interestingly, we found that such decline in anti-SARS-CoV-2 IgA levels was greater in younger individuals, though elderly people still displayed a relatively lower titer. Altogether, these findings may hence provide a possible explanation to the observed decreased efficacy of COVID-19 vaccines at preventing SARS-CoV-2 infection at 6 months after vaccination $[3,4]$, and 
prompt us to suggest that further studies should be planned to define whether targeted administration of vaccine boosters should be prioritized in subjects displaying faster decline of anti-SARS-CoV-2 IgA, in order to further limit infection and viral circulation within the population.

\section{Funding sources}

None.

\section{Declaration of Competing Interest}

The authors declare that they have no known competing financial interests or personal relationships that could have appeared to influence the work reported in this paper.

\section{Ethical approval}

All study subjects provided written informed consent for being vaccination and for undergoing anti-SARS-CoV-2 antibodies monitoring. The study was conducted in accordance with the Helsinki Declaration and cleared by the Ethics Committee of Verona and Rovigo Provinces (3246CESC). All procedures performed in studies involving human participants were in accordance with the 1964 Helsinki declaration and its later amendments.

\section{Acknowledgement}

None

\section{References}

1. Honein MA, Christie A, Rose DA, Brooks JT, Meaney-Delman D, Cohn A, et al. Summary of Guidance for Public Health Strategies to Address High Levels of 
Community Transmission of SARS-CoV-2 and Related Deaths, December 2020. MMWR Morb Mortal Wkly Rep 2020;69:1860-1867.

2. Omrani AS, Tleyjeh IM. Which are the best coronavirus disease 2019 vaccines? Clin Microbiol Infect. 2021 Aug 21:S1198-743X(21)00469-9. doi: 10.1016/j.cmi.2021.08.012. Epub ahead of print.

3. Rosenberg ES, Holtgrave DR, Dorabawila V, Conroy M, Greene D, Lutterloh E, et al. New COVID-19 Cases and Hospitalizations Among Adults, by Vaccination Status - New York, May 3-July 25, 2021. MMWR Morb Mortal Wkly Rep 2021;70:1306-1311.

4. Nanduri S, Pilishvili T, Derado G, Soe MM, Dollard P, Wu H, et al. Effectiveness of Pfizer-BioNTech and Moderna Vaccines in Preventing SARS-CoV-2 Infection Among Nursing Home Residents Before and During Widespread Circulation of the SARS-CoV-2 B.1.617.2 (Delta) Variant - National Healthcare Safety Network, March 1-August 1, 2021. MMWR Morb Mortal Wkly Rep 2021;70:1163-1166.

5. Sterlin D, Mathian A, Miyara M, Mohr A, Anna F, Claër L, et al. IgA dominates the early neutralizing antibody response to SARS-CoV-2. Sci Transl Med 2021;13:eabd2223.

6. Manalac J, Yee J, Calayag K, Nguyen L, Patel PM, Zhou D, Shi RZ. Evaluation of Abbott anti-SARS-CoV-2 CMIA IgG and Euroimmun ELISA IgG/IgA assays in a clinical lab. Clin Chim Acta 2020;510:687-690.

7. Nicholson S, Karapanagiotidis T, Khvorov A, Douros C, Mordant F, Bond K, Druce J, et al. Evaluation of 6 Commercial SARS-CoV-2 Serology Assays Detecting Different Antibodies for Clinical Testing and Serosurveillance. Open Forum Infect Dis 2021;8:ofab239.

8. Evans SJW, Jewell NP. Vaccine Effectiveness Studies in the Field. N Engl J Med 2021;385:650-651. 
9. Lippi G, Henry BM. How will emerging SARS-CoV-2 variants impact herd immunity? Ann Transl Med 2021;9:585.

10. Varnaitė R, García M, Glans H, Maleki KT, Sandberg JT, Tynell J, et al. Expansion of SARS-CoV-2-Specific Antibody-Secreting Cells and Generation of Neutralizing Antibodies in Hospitalized COVID-19 Patients. J Immunol 2020;205:2437-2446.

11. Lapić I, Šegulja D, Rogić D. Assessment of salivary antibody response to BNT162b2 mRNA COVID-19 vaccination. J Med Virol 2021;93:5257-5259.

12. Lippi G, Mattiuzzi C. Clinical value of anti-SARS-COV-2 serum IgA titration in patients with COVID-19. J Med Virol 2021;93:1210-1211. 
Figure 1. Kinetics of serum anti-spike S1 subunit IgA in 100 consecutive SARS-CoV-2 seronegative healthcare workers receiving a full cycle of Pfizer/BioNTech mRNAbased vaccination. The two arrows indicate the 2 vaccine doses administration.

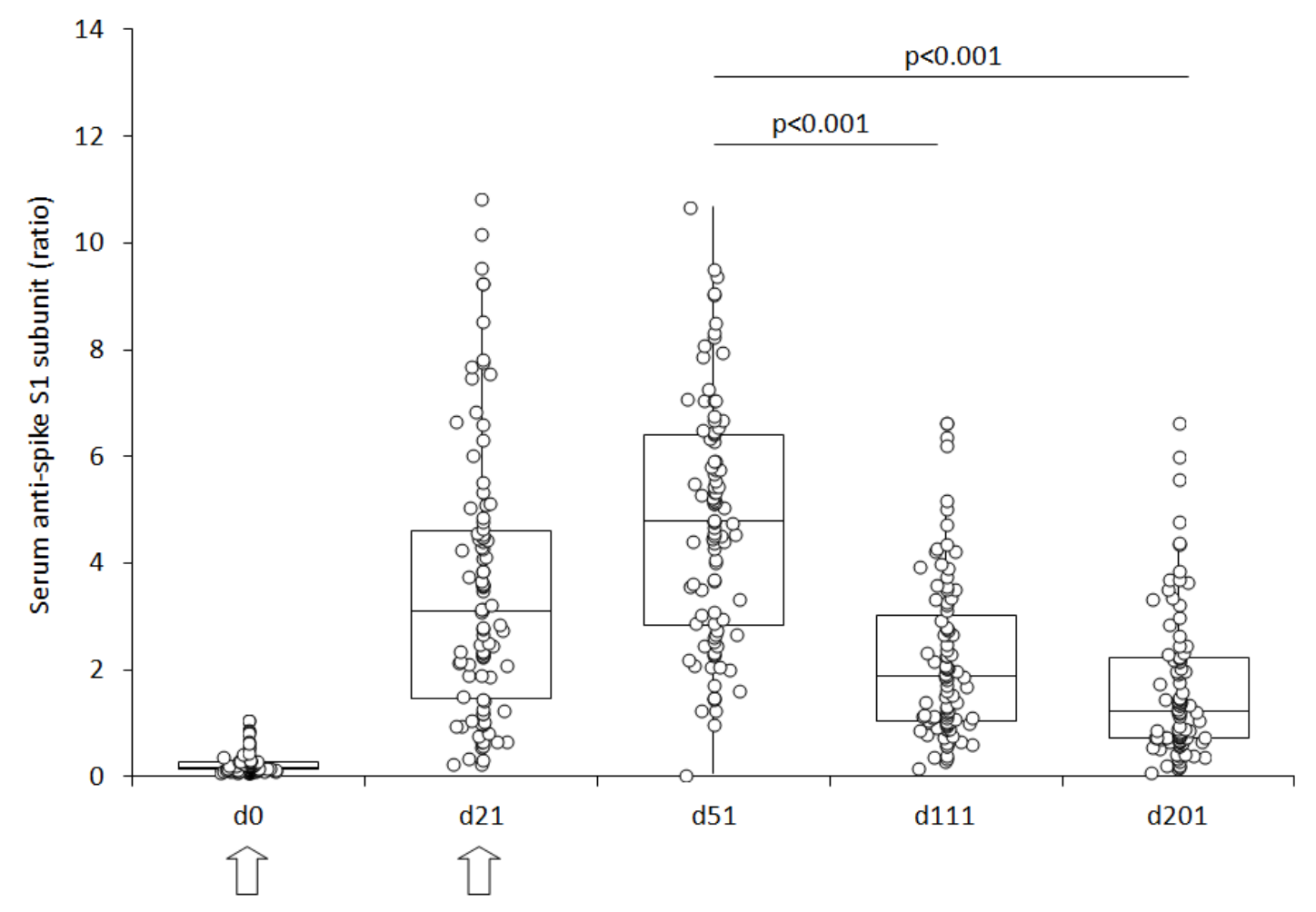


Figure 2. Spearman's correlation between the ratio of anti-spike S1 subunit IgA decline at 6 months compared to the peak (i.e., [201-day/51-day concentration]) and the age of vaccine recipients.

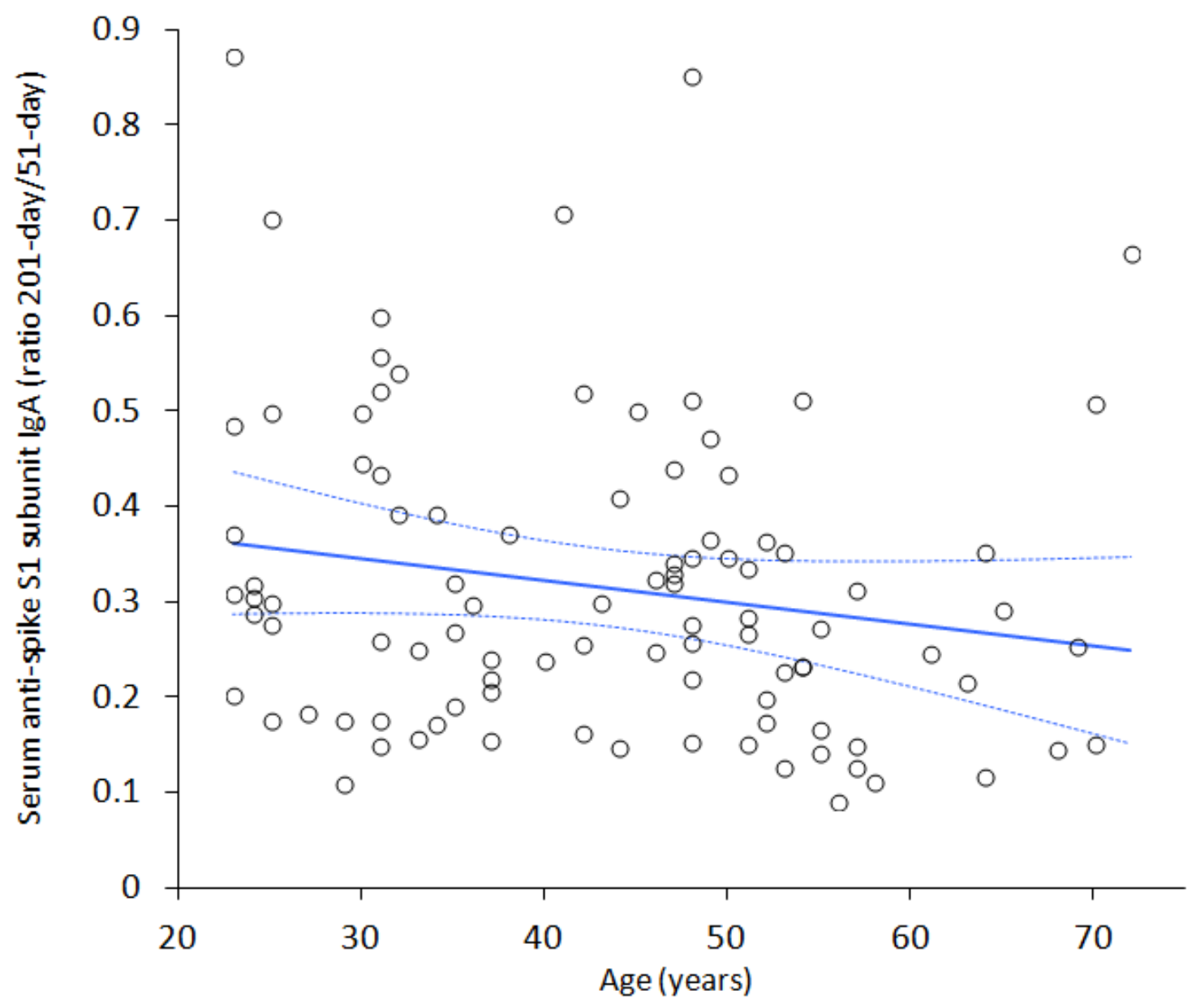

